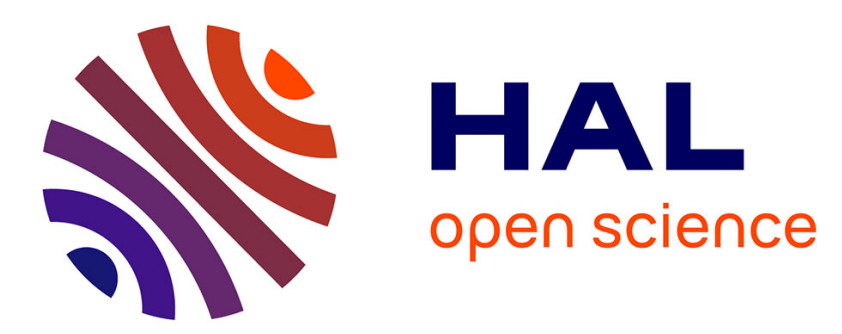

\title{
Towards a better understanding of the CMUTs potential for SHMapplications
}

Pauline Butaud, Patrice Le Moal, Gilles Bourbon, Vincent Placet, Emmanuel Ramasso, Benoît Verdin, Eric Joseph

\section{> To cite this version:}

Pauline Butaud, Patrice Le Moal, Gilles Bourbon, Vincent Placet, Emmanuel Ramasso, et al.. Towards a better understanding of the CMUTs potential for SHMapplications. Sensors and Actuators A: Physical , 2020, 313, pp.112212 (11). hal-03007448

\section{HAL Id: hal-03007448 https://hal.science/hal-03007448}

Submitted on 16 Nov 2020

HAL is a multi-disciplinary open access archive for the deposit and dissemination of scientific research documents, whether they are published or not. The documents may come from teaching and research institutions in France or abroad, or from public or private research centers.
L'archive ouverte pluridisciplinaire HAL, est destinée au dépôt et à la diffusion de documents scientifiques de niveau recherche, publiés ou non, émanant des établissements d'enseignement et de recherche français ou étrangers, des laboratoires publics ou privés. 


\title{
Towards a better understanding of the CMUTs potential for SHM applications
}

\author{
Pauline Butaud, Patrice Le Moal, Gilles Bourbon, Vincent Placet, \\ Emmanuel Ramasso, Benoit Verdin, Eric Joseph \\ Univ. Bourgogne Franche-Comté FEMTO-ST, CNRS/UFC/ENSMM/UTBM, \\ Department of Applied Mechanics 24, Chemin de l'Epitaphe 25000 Besançon - FRANCE
}

\begin{abstract}
The ability of Capacitive Micromachined Ultrasonic Transducer (CMUTs) to design broadband sensors for Structural Health Monitoring (SHM) is studied through both multi-frequency and bandwidth aspects. Elementary cells are composed of circular membranes fabricated using the standard MUMPS Process. The multi-frequency aspect, which involves different individual membranes from $50 \mu \mathrm{m}$ to $250 \mu \mathrm{m}$ radius, is theoretically addressed through a numerical modeling. The targeted frequency range, consistent with the SHM application, is then between $80 \mathrm{kHz}$ and $2 \mathrm{MHz}$. Geometrical features induced by the manufacturing process greatly affect the dynamic properties of the membranes and this is experimentally validated. The bandwidth aspect is also addressed on an array of identical $100 \mu \mathrm{m}$ radius membranes thus involving their intrinsic capabilities. Harmonic excitation with targeted frequencies $300 \mathrm{kHz}, 530 \mathrm{kHz}$ and $800 \mathrm{kHz}$, below and beyond the resonance frequency of the membranes, are performed. The influence of the bias voltage $\mathrm{V}_{D C}$ on the signal-to-noise ratio is studied according to the excitation frequency. As a result, a signal-to-noise of $20 \mathrm{~dB}$ is achieved around the resonance frequency. Finally, the circular membranes array is tested for acoustic emission sensing through a pencil lead break test. In spite of a low signal-tonoise ratio, acoustic events are clearly detected. The multi-frequency aspect and the large bandwidth capability of the CMUTs are hence demonstrated and highlight the adaptability of the sensor to its environment.
\end{abstract}

Keywords: MEMS, CMUT, SHM, experimental, modeling, circular membranes array 


\section{Introduction}

The Acoustic Emission technique which detects stress waves released as structural damage occurs is one of the Structural Health Monitoring (SHM) promising possibilities [1]. The development of a SHM system involves the enhancement of the data processing and consequently a good knowledge of the behaviour of the sensors used to record events. Different technologies are proposed for this purpose: optical fiber [2, 3], piezoelectric sensor [4, MEMS transducers as PMUTs (Piezoelectric Micromachined Ultrasonic Transducers) [5] and CMUTs (Capacitive Micromachined Ultrasonic Transducers) [6, 7, 8]. Our paper will be focused on the latter.

More than ten years ago, Ozevin et al. 9] highlighted the potential of the CMUTs transducers for Acoustic Emission (AE) measurements for SHM. The authors studied a MEMS chip containing multiple resonance capacitive-type vibration transducers and demonstrated the possibility to detect acoustic emission events associated to crack initiation and growth. The only drawback emphasized for this technology was the poor signal-to-noise ratio, which could be improved, according to the authors, by a better packaging and shielding, or by increasing the number of cells. The transducers proposed at this time were designed using the poly-MUMPS surface micromachining process [10]. Since then, other designs of CMUT have been proposed: a 3-D CMUT collocating two in-plane sensors and one out-of-plane sensor at the millimeter scale [11], a polymer-based CMUT [12] produced using micro-stereolithography, capacitive MEMS sensors manufactured with the electroplating method using the MetalMUMPs [13], all with promising properties, in particular a really interesting electromechanical coupling, and finally a signal-to-noise ratio suitable for the targeted applications. Nevertheless, as CMUTs supposedly required high bias voltage, this technology is less and less studied and PMUTs take the center stage [14].

CMUTs, especially in the context of SHM applications, still suffer from a lack of data and knowledge on their electromechanical dynamic behaviour from a theoretical and experimental point of view. The often recent and not yet fully mature micro-fabrication technologies used for their manufacture, and the multi-physical couplings at the various interfaces, are probably the main causes of this finding.

This article therefore aims to provide some answers to the ability of CMUTs to design broadband sensors for SHM applications. This mainly concerns two aspects: 
- the multi-frequency aspect involving different individual membranes (theoretically addressed and experimentally validated) ;

- the bandwidth aspect including the intrinsic capabilities of an array of identical membranes (approached from a purely experimental angle).

In the future, the two aspects could be combined to benefit from each other but, as a first step towards this goal, the present work examines them separately.

For this purpose, a chip composed of CMUT unit membranes with different radii from $50 \mu \mathrm{m}$ to $250 \mu \mathrm{m}$ and CMUT arrays of $100 \mu \mathrm{m}$ radius cells is designed. The target frequency range is between $80 \mathrm{kHz}$ and $2 \mathrm{MHz}$. The manufacturing process, the constitutive materials and the key points of the geometries are outlined in this paper. Besides, in a numerical study, the impact of the geometry resulting from micro-fabrication technologies, and more particularly the influence of the fabrication process "Multi-Users MEMS Processes" (MUMPS) on the resonance frequency of CMUTs are studied. Indeed some geometrical elements are often ignored in the literature with regard to the effects of residual stresses and squeeze film damping. Residual stresses ranges are identified and briefly discussed on the basis of theoretical and experimental matching of resonance frequencies. However, the squeeze film damping effects will be neglected in this study due to the particular design of our CMUT membranes. Finally the pull-in voltage is determined for each different radius membrane.

Furthermore an experimental study on the CMUT membranes is conducted. The chip is integrated in a brass package to minimize parasitic effects. First of all, the resonance frequencies of the different radius membranes are measured to validate the design and the multi-frequency abilities of CMUTs. Then, the array of $100 \mu \mathrm{m}$ radius membranes is experimentally characterized. The influence of the DC bias voltage on the sensitivity of the CMUTs array is highlighted through a single frequency excitation. The CMUT ability to respond to a frequency range below and beyond its resonance frequency is illustrated. Finally, the capacity of the array to detect AE events is demonstrated by a broadband excitation obtained by a pencil lead break; this despite a low sensitivity and a signal-to-noise ratio to be improved.

The paper is organized as follows. Section 2 outlines the design of the CMUT chip. Section 3 presents the numerical study of unit cells. Section 4 illustrates the CMUT membranes properties within the application SHM 
framework through experimental validation. In the last section, conclusions are drawn on the potential of CMUTs for SHM applications with regard to the two aspects considered, i.e. multi-frequency and bandwidth.

\section{Sensor design}

A CMUT sensor usually comprises several elementary capacitive micromachined ultrasonic cells acting as microphone membranes. The cell membranes are vibrating subjected to ultrasonic waves from the surrounding medium, either fluid or solid. In the present case, mechanical solid or surface waves propagating in monitored structures are transferred to the cells through the membrane clamping supports. Sensing the ultrasonic waves is achieved by converting vibration-induced membrane displacements into electrical energy via capacitive effects. The generic architecture, the mechanical properties and the geometrical key elements of the CMUT cells are described in this section.

The CMUT unit cells, based on perforated circular membranes clamped at periphery, are fabricated by surface micromachining using the standard fabrication process "Multi-User MEMS Processes" (MUMPS, run \#120). The top and sectional views of an elementary CMUT cell layout, as proposed in Figure 1, show the successive constituent layers:

- a silicon nitride layer on the silicon handle substrate isolating both bottom and top electrodes,

- a first polysilicon layer which is patterned according to the ground, feed electrodes and electric tracks,

- two sacrificial silicon oxide layers which are afterwards removed to create the electrostatic gap,

- a second polysilicon layer constituting the vibrating membrane which clamping periphery contacts the feed electrode.

Once the second polysilicon layer is deposited, membranes, clamping parts and several holes are patterned by Reactive Ion Etching [15]. The holes are etched to release the vibrating membrane, which becomes suspended by dissolving the sacrificial oxide layers underneath with hydrofluoric acid. MUMPS design rules [16, 17] recommend to design holes with a minimum diameter of $4 \mu \mathrm{m}$ with $30 \mu \mathrm{m}$ pitch. In our work, holes enlarged to $10 \mu \mathrm{m}$ 


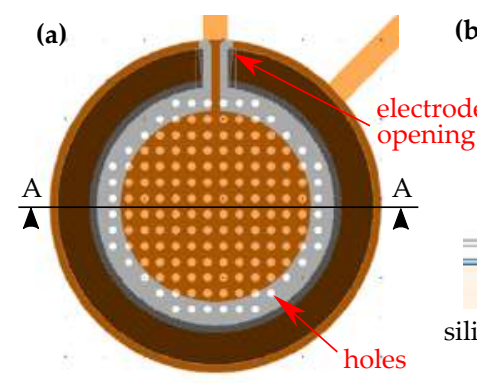

(b)

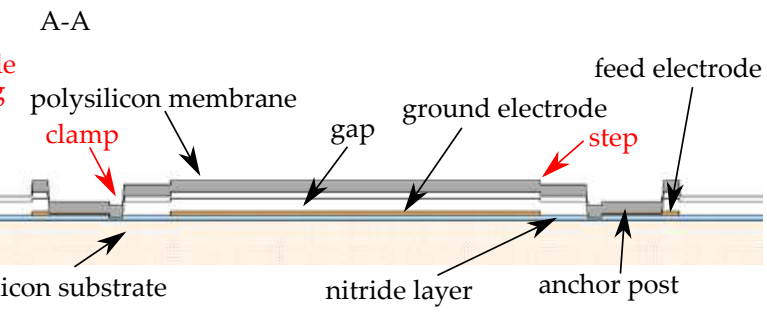

Figure 1: (a) Top and (b) sectional views of a typical membrane layout

diameter with reduced $20 \mu \mathrm{m}$ pitch are preferred in order to control in the design phase the minimization of the squeeze film stiffening of the air layer in the cavity.

Mohite et al [18] introduces the notion of an equivalent pressure cell depending on the hole configuration (staggered with a hexagonal pattern or non-staggered with a square pattern) which radius is roughly 0.5 times the pitch. In this case, the radius of the pressure cell around $10 \mu \mathrm{m}$ leads to a squeeze number lower than 0.5 for all the $50 \mu \mathrm{m}$ to $250 \mu \mathrm{m}$ radius membranes. Referring to works of Galisultanov et al [8] based on solutions developed by Crandall et al [19], open cavity CMUTs for such very low squeeze number are strained by negligible elastic force from the compressed air layer. Meanwhile, open cavity CMUTs reveal higher damping forces that could contribute to wider bandwidth. On the other hand, holes ratio cannot be too much increased, because the CMUT capacitance would decrease and consequently the sensing sensitivity as well.

Our design based on $5 \times 5 \mathrm{~mm}^{2}$ chips includes single elementary CMUT cells for the frequency response and pull-in voltage determination, and cell arrays for the sensor tests under harmonic and broadband excitation. The thickness of the polysilicon membrane (so-called "Poly 2" in [16]) and the electrostatic gap formed by the silicon oxides etching (so-called "Oxide 1 and Oxide 2" in [16]) are experimentally measured by profilometry respectively around $1.30 \mu \mathrm{m}$ and $2.40 \mu \mathrm{m}$.

The radius of the fabricated CMUT cells are: $50 \mu \mathrm{m}, 75 \mu \mathrm{m}, 100 \mu \mathrm{m}$, $150 \mu \mathrm{m}, 200 \mu \mathrm{m}$ and $250 \mu \mathrm{m}$ and the measured thickness about $1.30 \mu \mathrm{m}$ allowing a frequency range between $80 \mathrm{kHz}$ and $2 \mathrm{MHz}$ to be swept. This frequency domain is consistent with the AE scope usually involving transient signals in the $100 \mathrm{kHz}-1 \mathrm{MHz}$ range. 

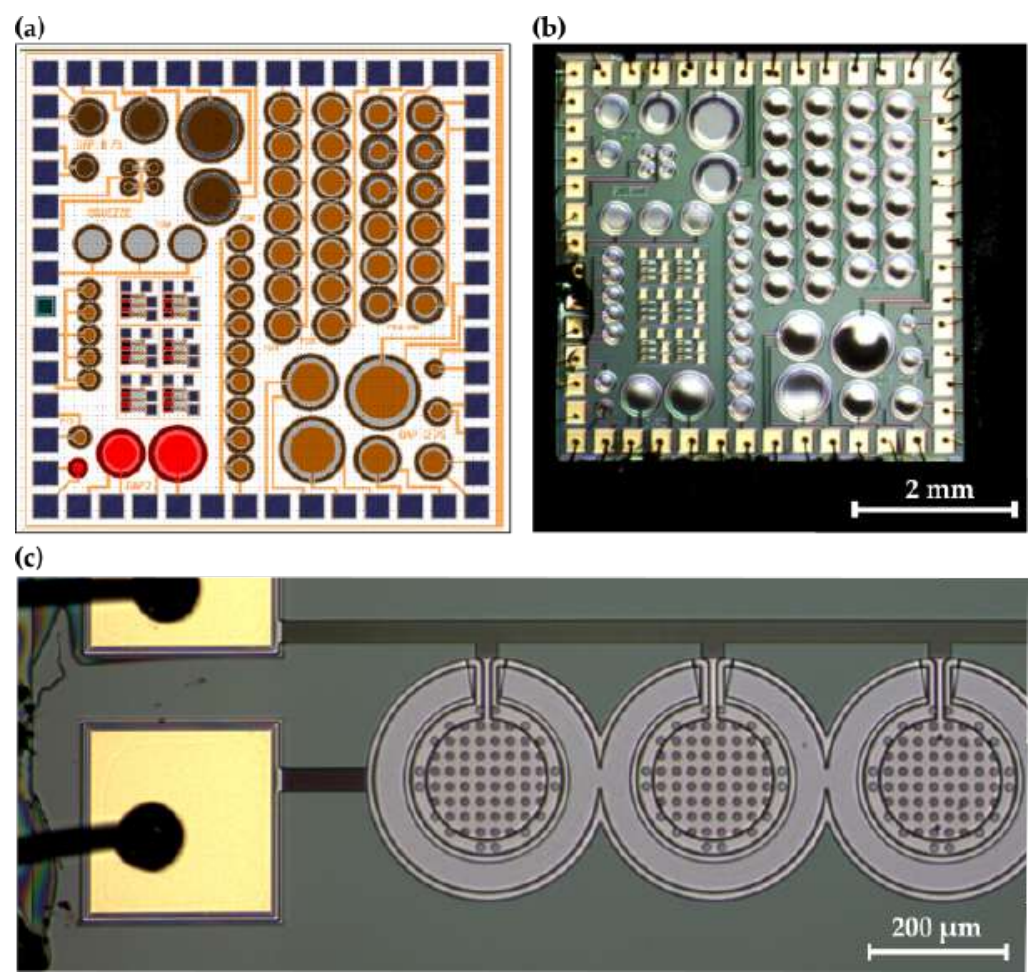

Figure 2: Design of the CMUT sensor: (a) layout and (b) fabricated chip, (c) electrical connections and top view of a $100 \mu \mathrm{m}$ radius membranes array.

Figure 2(a) and Figure 2(b) show respectively the layout and the fabricated chip with elementary CMUT cells at the top left and the bottom right side and an array of 9 membranes of $100 \mu \mathrm{m}$ radius at the middle (studied in section 4). On the photography of Figure 2(b), color gradients indicates that some membranes have a deformed shape after fabrication resulting from unexpected residual stresses. As expected, the deformed shapes are greater as the membrane radius is larger. Figure 2(c) focuses on the electrical connections and a top view of a $100 \mu \mathrm{m}$ radius perforated circular membrane array.

The manufacturing process and the operating specifications induce four geometric structural features which could potentially impact the frequency response of the CMUT cell. Firstly, a step (marked "step" in Figure 1(b)) in the radial cross-section of the membrane due to the transfer of the ground electrode pattern modifies the membrane stiffness. Secondly, the clamp- 
ing conditions (marked "clamp" in Figure 1(b)) are not perfect due to the sidewall link between the membrane and the anchor posts. Thirdly, holes (marked "holes" in Figure 1(a)), patterned in the CMUT cell for the releasing of the suspended membrane, reduce the mass and the stiffness of the polysilicon membrane. Lastly, an opening (marked "electrode opening" in Figure 1(a)) in the annular clamping walls, needed for the connection between the electric track and the ground electrode under the membrane, affects the clamping conditions. The next section is dedicated to the study of those features by a numerical study.

\section{Numerical modelling}

The frequency response (resonance frequency, bandwidth and maximal vibration amplitude) of the elementary cells determines the sensor characteristics. Controlling the bandwidth and the maximal vibration amplitude is very challenging depending on different loss mechanisms (air loss, support loss, thermomechanical loss) [20, 21, 22]. On the other hand, the resonance frequency can be accurately defined by the geometrical dimensions, the mechanical properties of the constitutive material, the clamping conditions and the bias DC voltage for electrostatic devices.

The "stepped geometry", the "real boundary conditions", the "releasing holes" and the "electrode opening" directly involved by the surface micromachining steps are successively and accurately analyzed by finite elements simulations with ANSYS ${ }^{\circledR}$ in terms of resonance frequencies. Then the pullin voltage is determined for each different radius membrane because it is an essential electromechanical parameter conditioning the operating range in terms of the DC bias voltage itself determining the sensor sensitivity.

\subsection{Geometric key elements modelling}

Leissa [23] develops closed-form solutions for mode shapes and resonance frequencies of circular plates. The resonance frequency is expressed as a function of geometric dimensions $R$ and $h$ (respectively the radius and the thickness of the membrane), of mechanical properties $E, \nu$ and $\rho$ (respectively the Young's modulus, the Poisson's ratio and the density of polysilicon material) and of a parameter $\lambda$ depending on the mode shapes and the boundary 
conditions:

$$
f=\frac{\lambda^{2}}{2 \pi R^{2}} h \sqrt{\frac{E}{12 \rho\left(1-\nu^{2}\right)}} .
$$

The CMUT circular cells involve the first mode shape and are clamped at the periphery. Thus, the parameter $\lambda$ is around 10.2158. Moreover, MUMPS fabrication capabilities lead to consider $h=1.30 \mu \mathrm{m}, E=160 \mathrm{MPa}, \nu=0.22$ and $\rho=2330 \mathrm{~kg} / \mathrm{m}^{3}$. As reported in Table 1 , membrane radii from $50 \mu \mathrm{m}$ to $250 \mu \mathrm{m}$ determine resonance frequency from $80 \mathrm{kHz}$ to $2 \mathrm{MHz}$.

The top and sectional views of the membrane (Figure 1) present the geometry of each fabricated CMUT cell showing some significant discrepancies with the analytical approach assumptions. As previously described in the manufacturing process section, four key discrepancies can be noticed: a stepped geometry, non-optimal clamping conditions at the periphery, perforated membranes and an opening in the clamping ring. FEM modal analyses with the ANSYS ${ }^{\circledR}$ finite element software are performed considering accurate $3 D$ models meshed by 20 node hexahedral SOLID186 elements. The successive models take into account an additional key discrepancy in order to point out the influence of each geometrical particularity.

First of all, an initial benchmark model based on a quarter of the structure allows to validate at the same time the analytic results and the meshing size of the FEM model. The results in terms of the first resonance frequency are identical and not reported in the following.

Stepped geometry. This modelling includes the stepped geometry of the membrane (Figure 3). It shows a stiffening of the structure involving an increasing

\begin{tabular}{lcccccc}
\hline Radius $[\mu \mathrm{m}]$ & 50 & 75 & 100 & 150 & 200 & 250 \\
\hline Analytic results $[\mathrm{kHz}]$ & 2073 & 921 & 518 & 230 & 130 & 83 \\
Stepped geometry model $[\mathrm{kHz}]$ & 2202 & 984 & 555 & 247 & 139 & 89 \\
Boundary conditions model $[\mathrm{kHz}]$ & 2154 & 969 & 548 & 245 & 138 & 89 \\
Releasing holes model $[\mathrm{kHz}]$ & 2111 & 931 & $515-517$ & $229-230$ & $128-128$ & $82-84$ \\
Electrode opening model $[\mathrm{kHz}]$ & 2074 & 924 & 516 & 229 & 128 & 84 \\
\hline
\end{tabular}

Table 1: Resonance frequency according to the radius of the membrane, estimated analytically and by numerical simulation. 
of the resonance frequency around $7 \%$ which is, until now, rarely, if ever, commented about surface micro machined architectures according to the authors. The higher the radius, the stiffer the structure is, with a slight variation between $6.3 \%$ and $7.6 \%$ for the $50 \mu \mathrm{m}-250 \mu \mathrm{m}$ radius range (Table 1).

(a)

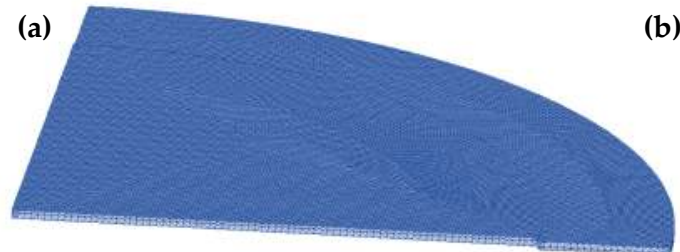

(b)

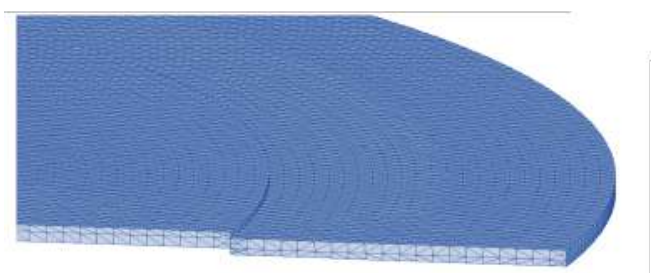

Figure 3: Structural meshing for the "stepped geometry" model configuration: membrane thickness modified by the Poly 0 step, (a) complete and (b) zoom.

Real boundary conditions. This modelling considers the specific clamping conditions (Figure 4). It introduces a softening of the structure leading to a slight decrease in resonance frequencies, decrease of maximum $2.2 \%$ for the radius of $50 \mu \mathrm{m}$. The higher the radius, the less the clamping conditions have to be taken into account. For example, the variation of the resonance frequency for the $250 \mu \mathrm{m}$ radius is at $0.50 \%$ (Table 1 ).

(a)

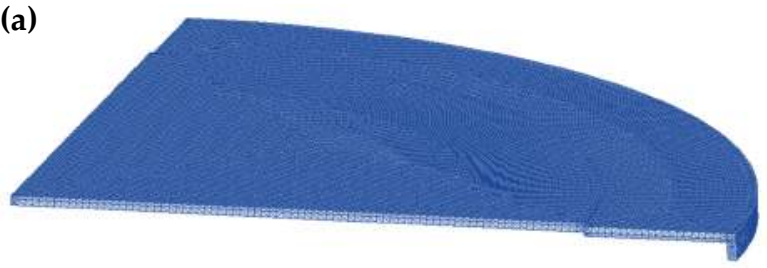

(b)

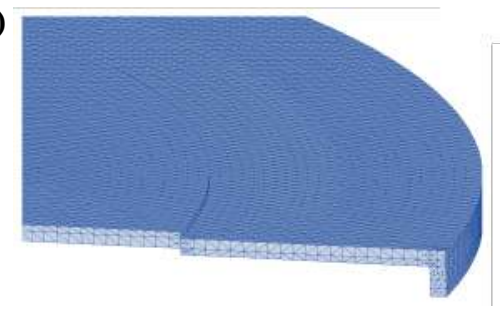

Figure 4: Structural meshing for the "real boundary conditions" model configuration: clamping conditions via the peripheral sidewall, (a) complete and (b) zoom.

Releasing holes. This modelling involves a perforated membrane as it can be seen in the Figure 5. It highlights a significant softening of the structure: the relative decrease in resonance frequencies varies from $2.0 \%$ to $7.7 \%$ for a radius between $50 \mu \mathrm{m}$ and $250 \mu \mathrm{m}$ (Table 1). This relative decrease of resonance frequencies is all the more remarkable as it results from two opposite contributions: one tends to increase them by reducing the mass of the 
membrane varying from $12 \%$ to $19 \%$, and the other tends to decrease them by reducing the stiffness of the membrane. Thus, the holes in the membranes seem to induce a quite significant reduction of the stiffness membranes.
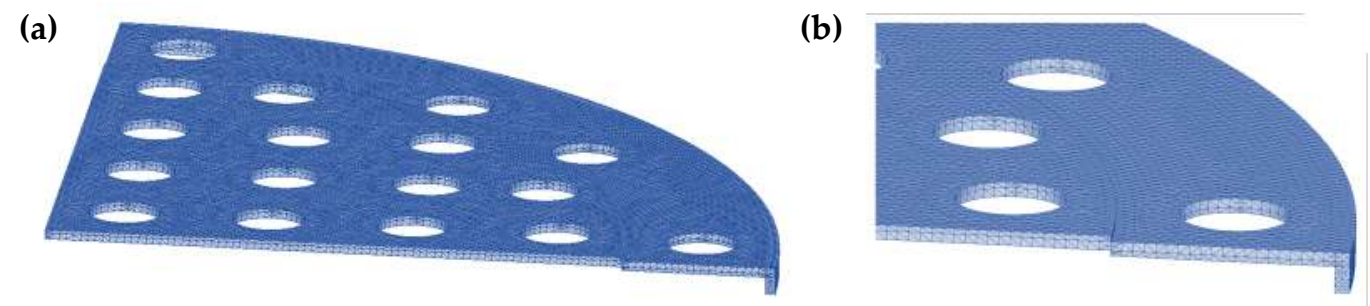

Figure 5: Structural meshing for the "releasing holes" model configuration: perforated membrane, (a) complete and (b) zoom.

Electrode opening - complete model. Lastly, the modelling takes into account the opening in the annular clamping and therefore includes the complete structure due to the symmetry breakage (Figure 6). Due to time-consuming $3 \mathrm{D}$ numerical simulations, this model requires, compared to the previous models, a very slight worsening in mesh size for radius above $75 \mu \mathrm{m}$. Practically, the size element is increased by $1 \mu \mathrm{m}(100 \mu \mathrm{m} / 150 \mu \mathrm{m}: 1 \mu \mathrm{m}$ to $2 \mu \mathrm{m}$, $200 \mu \mathrm{m}$ : $2 \mu \mathrm{m}$ to $3 \mu \mathrm{m}, 250 \mu \mathrm{m}: 3 \mu \mathrm{m}$ to $3.9 \mu \mathrm{m})$. Therefore, the resonance frequency variations for the highest radius are partly linked to the meshing differences. In order to accurately evaluate the influence of the opening in the annular clamping, the "releasing holes" model is also run with the same worsened meshing (cf. right result values in the line "releasing holes model" in Table 1). In this case, the intrinsic influence is clearly proved showing a slight decreasing of the resonance frequency between $0.10 \%$ and $1.7 \%$ in the radius range.

To conclude on this numerical study, two geometrical configurations, i.e. the boundary conditions and the opening in the annular clamping, can be reasonably neglected with very small influences on the first resonance frequency (below 2\%). These influences are all the more negligible the larger the radius of the membrane is. On the other hand, the stepped geometry and the perforated membranes respectively lead to significant stiffening and softening of the structure with relative variations of up to $8 \%$ for the first resonance frequency. Overall, these effects cancel each other out in this case, 

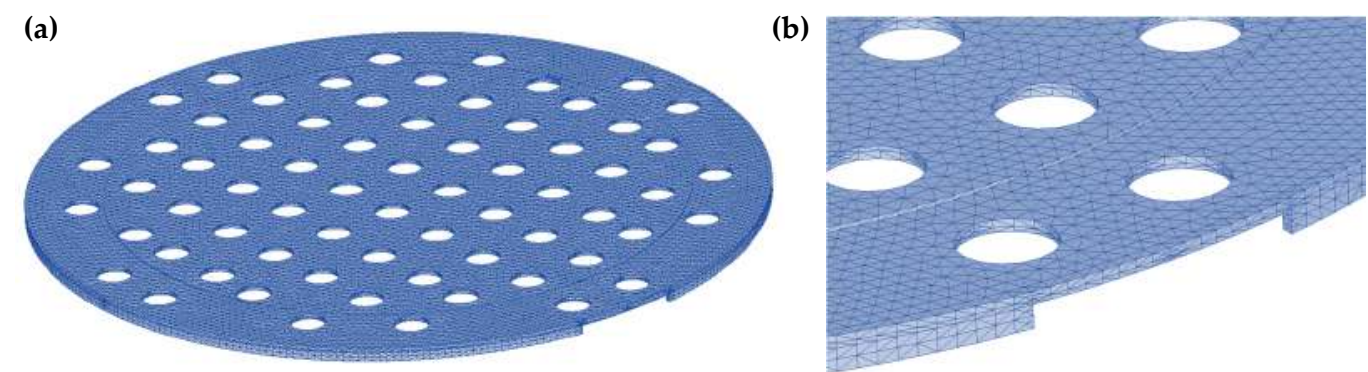

Figure 6: (a) Complete model of the membrane and (b) a zoom on the electrode opening in the annular clamping.

but reliable design requires careful consideration. Indeed, it should be different if the parameters of the stepped geometry and perforated membranes are changed: for example, the radius and thickness of the bottom ground electrode, thickness of the membrane and holes density.

\subsection{Pull-in voltage}

Another key point for the membrane design is the identification of the pull-in voltage corresponding to the membrane collapse. The pull-in voltage determines the operating range in terms of the DC bias voltage of the sensor: indeed no insulating layer protects the bottom ground electrode from the top supplied electrode (i.e. the membrane itself). The sensing parameters here are the electrostatic charges or electrical current which is directly proportional to the DC bias voltage. As the previous dynamic study, the complete model is used to discriminate the static influence of realistic structure geometries on the pull-in voltage. Static analyses are performed using the electromechanical line transducer element TRANS126 in ANSYS ${ }^{\circledR}$. These elements are generated from the bottom face of the perforated membrane and more particularly under the Poly 0 step which is the image of the bottom ground electrode. However, such elements do not intrinsically include edge effects. Figure 7(a) shows a simplified model with only a few TRANS126 elements to maintain readability. Identification of the pull-in voltage is achieved incrementally with $0.1 \mathrm{~V}$ steps. Figure 7(b) illustrates the deformation of a $100 \mu \mathrm{m}$ radius membrane just beyond the pull-in voltage. To do so, a "virtual" insulating layer of $0.05 \mu \mathrm{m}$ is added for convergence convenience. Table 2 reports numerical pull-in voltages from finite element simulations to be compared with the analytical values calculated for a circular, perfectly clamped, uniform, and non-perforated membrane on the basis 
of the approach described by Nikoozadeh et al [24].

As can be seen, numerical and analytical results are very similar, fully confirming the findings of the dynamic study. In dynamics, numerical and analytical resonance frequencies result from stiffening of "the stepped geometry", softening of "the perforated membrane", and from the reduction of the membrane mass. In the same way, numerical and analytical pull-in voltages result from the same two effects of stiffening and softening and from reduction of resulting electrostatic forces. Indeed, while holes in dynamics reduce the membrane mass, they also reduce in statics the opposing electrode surfaces and thus the resulting electrostatic forces by the same proportion.

(a)

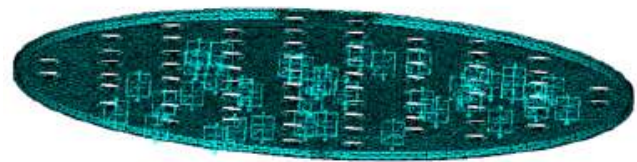

(b)
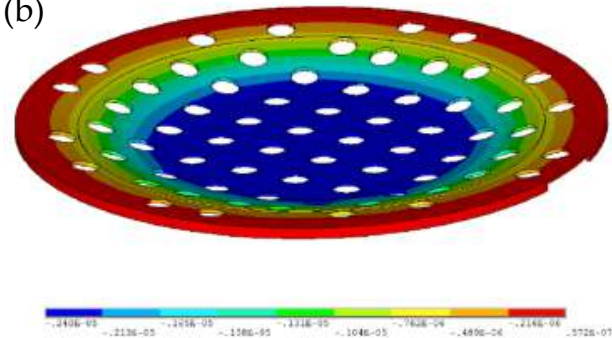

Figure 7: (a) Mesh of the model with TRANS126 elements and (b) the deformation of a $100 \mu \mathrm{m}$ radius membrane at the pull-in voltage (around $116 \mathrm{~V}$ ).

\begin{tabular}{lcccccc}
\hline Radius $[\mu \mathrm{m}]$ & 50 & 75 & 100 & 150 & 200 & 250 \\
\hline Analytic pull-in voltage $[\mathrm{V}]$ & 466.6 & 207.3 & 116.5 & 51.6 & 28.6 & 18.3 \\
Numerical pull-in voltage [V] & 465.8 & 207.6 & 115.5 & 51.3 & 28.6 & 18.7 \\
\hline
\end{tabular}

Table 2: Pull-in voltage according to the radius of the membrane of interest.

The following section, as this one from a numerical point of view, is focused on determining the electromechanical characteristics of CMUT based on an experimental tests campaign.

\section{Experimental characterizations}

\subsection{Resonance frequency of the membranes}

The first step of the experimental campaign is to check the validity of the manufacturing process by measuring the resonance frequency of the membranes and to compare it with the numerical model. At this stage, the 
electromechanical coupling is not taking into account, i.e the response of a membrane is captured through the displacement of the membrane and not with the electrical signal generated by the MEMS.

Materials and methods. The CMUT chip is wire-bonded in a ceramic package and a probe station is used to supply the cell (Figure 8). Elementary CMUT cells are tested one after the other (at the bottom right in Figure2(a)). A bias voltage $V_{D C}$ (small compared to the pull-in voltage in order to avoid the electrostatic softening effect) is applied to the membrane terminals and a frequency sweep is performed with an alternating current $V_{A C}$ (small compared to $V_{D C}$ in order to avoid electrostatic non-linearities). A laser vibrometer (Figure 8) is directed at the center of the membrane to measure the velocity amplitude of the membrane according to the excitation frequency. The resonance frequency of the membrane is determined when the maximum of amplitude is reached. The measurements have been repeated on three different chips.

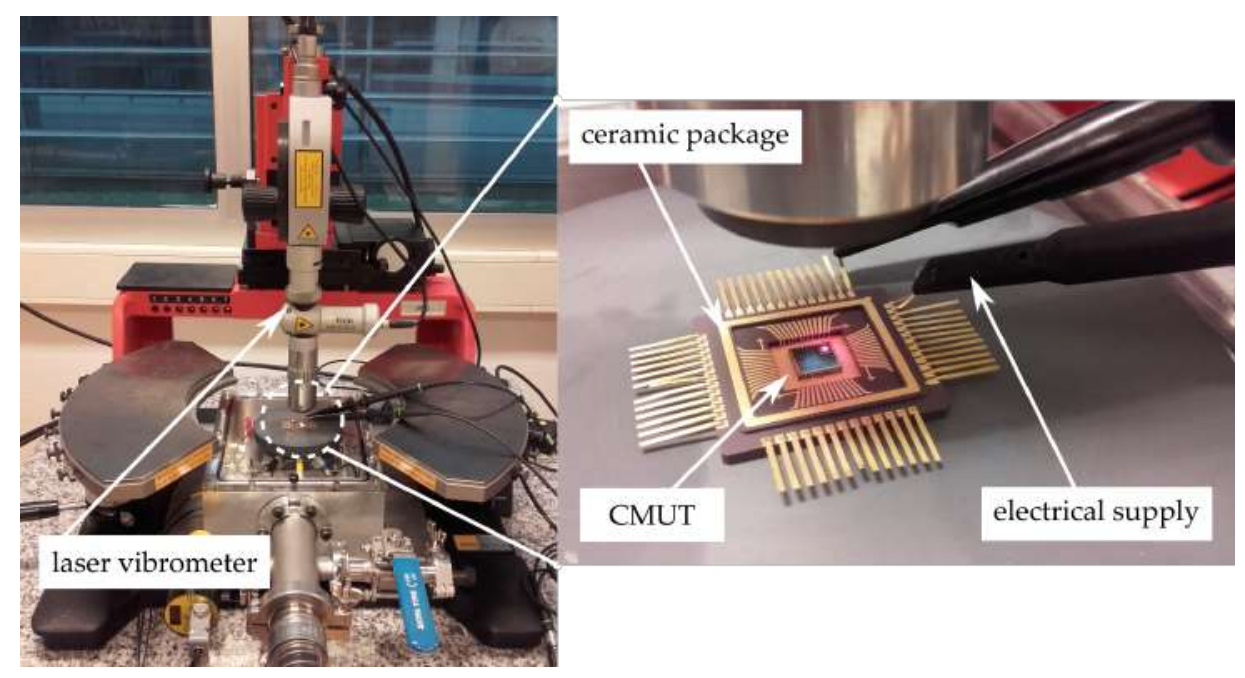

Figure 8: Experimental setup to measure the resonance frequency of membranes

Results. Table 3 synthesises the measurements. The experimental resonance frequencies are highly reproducible for radii up to $150 \mu \mathrm{m}$ with a maximal relative standard deviation around $2 \%$. The manufacturing process is then quite stable. In this radius range, the numerical resonance frequencies calculated from the complete model correctly approximate experimental ones by 


\begin{tabular}{l|cccccc}
\hline Radius $[\mu \mathrm{m}]$ & 50 & 75 & 100 & 150 & 200 & 250 \\
\hline Experimental $f_{0}[\mathrm{kHz}]$ & $1990 \pm 14$ & $854 \pm 6$ & $453 \pm 2$ & $228 \pm 4$ & $174 \pm 15$ & $139 \pm 28$ \\
Numerical $f_{0}[\mathrm{kHz}]$ & 2074 & 924 & 516 & 229 & 128 & 84 \\
Residual stress range $[\mathrm{MPa}]$ & $-12.5 /-9.0$ & $-9.5 /-8.0$ & $-8.0 /-7.0$ & $-1.0 / 0.5$ & $4.5 / 10.0$ & $4.5 / 17.0$ \\
\hline
\end{tabular}

Table 3: Resonance frequency $f_{0}$ according to the radius of the membrane, measured experimentally, compared to the complete model (without any residual stress) and calculated residual stress range for experimental and theoretical frequencies matching.

a relative excess of up to $12 \%$.

By introducing an initial stress state into the complete model, an average level of homogeneous residual stresses has been estimated for each radius by matching experimental and numerical resonance frequencies (Table 3). For radii less than $150 \mu \mathrm{m}$, the stress level is perfectly consistent with the values given by MUMPS (between $-20 \mathrm{MPa}$ and $0 \mathrm{MPa}$ with a nominal value at $-10 \mathrm{MPa}$ ). Beyond a radius of $150 \mu \mathrm{m}$, on the one hand, the measurements are not reproducible and, on the other hand, the calculated stress levels do not match the MUMPS range. This could be explained by membranes slender enough to induce critical buckling stresses in the MUMPS stress range (i.e. about $-14 \mathrm{MPa}$ and $-9 \mathrm{MPa}$ respectively for $200 \mu \mathrm{m}$ and $250 \mu \mathrm{m}$ radius). Thus, membrane buckling can lead to a redistribution of internal stresses by gradients into the thickness and to a modification of the structure (arch shape) inducing a dynamic stiffening of the membrane. This stiffening then results in the identification of a homogeneous initial tensile state.

To conclude, the correlation of experimental and theoretical resonant frequencies of moderate slenderness membranes (radius to thickness ratio below 150) demonstrates the validity of assumptions of the complete numerical model taking into account realistic residual stresses. Moreover, the experimental and theoretical multi-frequency aspect of membranes of different radii is highlighted by a broad covered frequencies range from $100 \mathrm{kHz}$ to $2 \mathrm{MHz}$.

\subsection{Electro-mechanical characterization}

In this section, the objective is to quantify the electrical response of a CMUT array of identical membranes to an external mechanical excitation at a specified frequency. The bias voltage influence is also studied. 


\subsubsection{Materials and methods}

The CMUT chip is integrated in a brass package as it can be seen in the Figure 9. The $5 \mathrm{~mm}$ square chip is surrounded by a PCB. The brass package is linked to the electric mass of the CMUT chip. This setup makes it possible to drastically reduce the electro-magnetic noise picked up by the cells. It can be noticed that the assembly is a $24 \mathrm{~mm}$ square, but on the underside 9(c), in contact with the test piece, a $10 \mathrm{~mm}$ square is in over-thickness in order to improve the contact between the sensor and the specimen.

Measurements are focused on the array of 9 membranes of $100 \mu \mathrm{m}$ radius (at the middle in Figure 2(a), and in Figure 2(c)) for which it is recalled that the resonant frequency and the pull-in voltage are about $450 \mathrm{kHz}$ and $116 \mathrm{~V}$ respectively. For the experiment, a Cooknell power supply SU3/C and an amplifier CA7/C (Figure 10) are used to power the CMUT chip and to measure the electrical charges generated by the membranes vibrations. The DC bias voltage applied on the CMUT is varying between $0 \mathrm{~V}$ and $60 \mathrm{~V}$ by 10 V step.
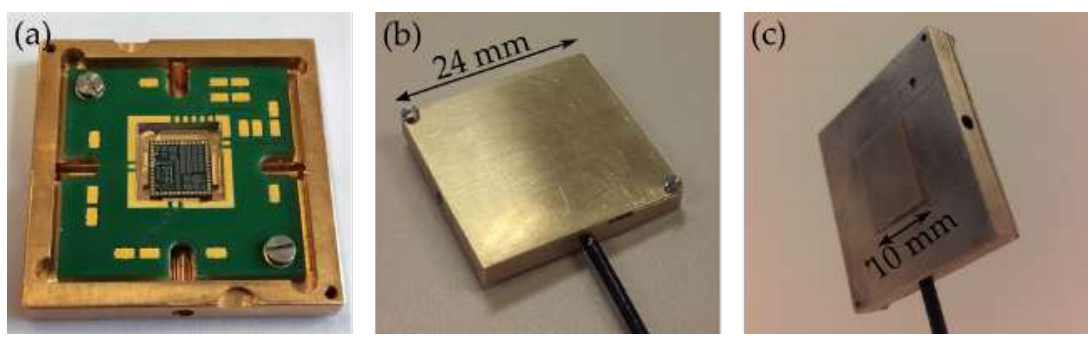

Figure 9: CMUT chip integrated in a brass package, (a) box open, (b) box closed - top view and (c) bottom view.

The specimen used is an aluminum beam with $200 \mathrm{~mm}$ length, $30 \mathrm{~mm}$ width and $3 \mathrm{~mm}$ thick, hanging by wires (Figure 10). The acoustic waves propagation through the beam is studied with two kinds of sensor: one is the CMUT, the other is a classical $\mu 80$-type sensor (Mistras Group Ltd.). Spring clamps are used to attach the sensors on one end of the beam. A permanent contact is obtained thanks to an industrial coupling gel which ensures a good transmission of the signal. A Picoscope $4824{ }^{\circledR}(5 \mathrm{MS} / \mathrm{s})$ is used to record the sensor signals. 


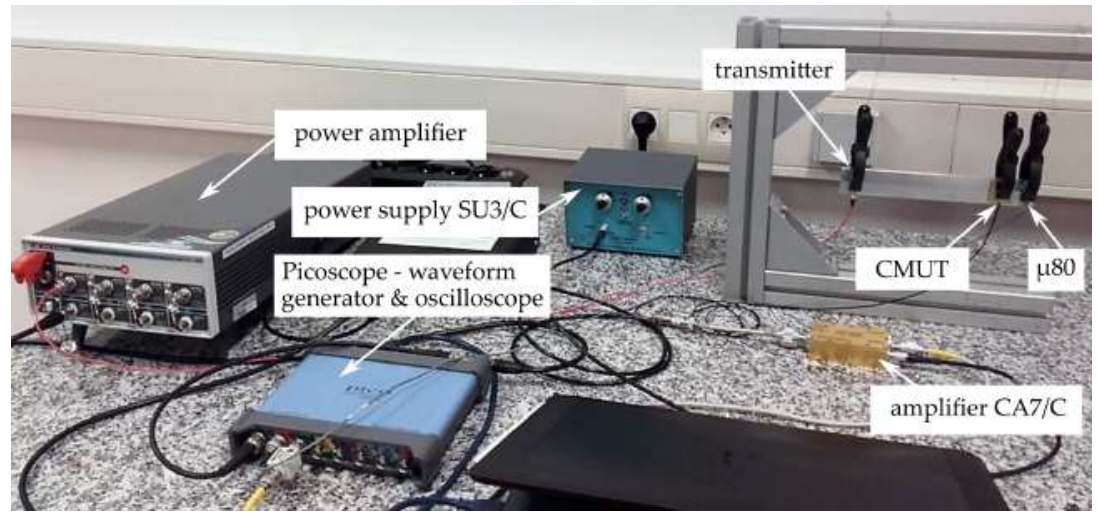

Figure 10: Experimental setup for harmonic excitation.

In order to control the frequency of the acoustic waves, a $\mu 80$ is clamped at the opposite end of the beam from the measurement (Figure 10). The $\mu 80$ transmitter (Mistras Group Ltd.) is first used at $300 \mathrm{kHz}$ which corresponds to the main resonance frequency of the sensor as it can be seen on its data sheet (Figure 11). The objective is to limit the impact of the transmitter on the excitation signal and to avoid interference frequencies on the excitation as much as possible. In addition, the $\mu 80$ is also used at $530 \mathrm{kHz}$, a frequency close to the resonance frequency of the studied CMUT, and at $800 \mathrm{kHz}$, on the plateau of the $\mu 80$, in order to map the behaviour of the CMUT over a wide frequency range. A 6-cycle sine wave windowed excitation is generated (amplitude $\pm 1 \mathrm{~V}$ ) with a Picoscope $4824^{\circledR}$ and amplified with a fixed gain of 50 (Tabor Electronics 9100 A).

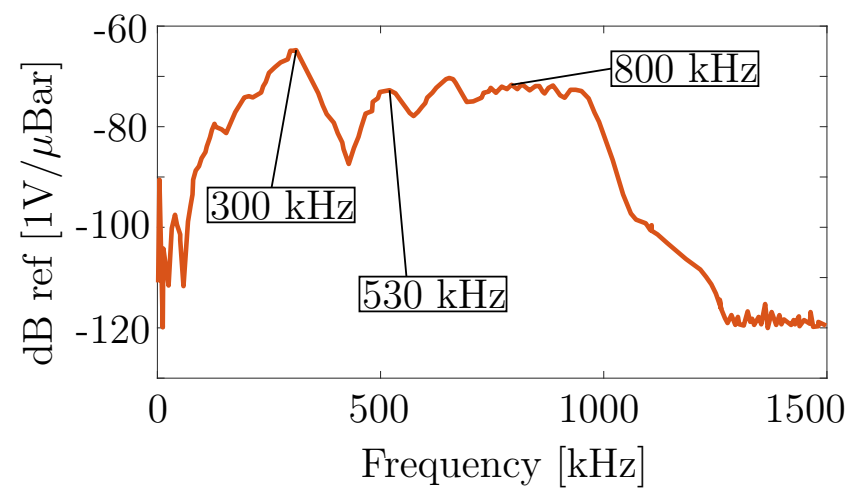

Figure 11: Frequency response of sensor $\mu 80$, data sheet from Mistras Group Ltd. 


\subsubsection{Results - harmonic excitation}

First of all, the raw signals for excitation at $530 \mathrm{kHz}$, which is close to the resonant frequency of our membranes, are studied. Figure 12(a) and (b) show the temporal signals measured respectively by the CMUT (with different bias voltage) and by the $\mu 80$. The reported amplitudes in $\mathrm{mV}$ are the image of the mechanical - electrical transduction carried out by the CMUTs and the $\mu 80$ sensor. Figure 12(c) and (d) show the FFT (Fast Fourier Transform) of the temporal signals.

Concerning the $\mu 80$, it can be noticed that not only the $530 \mathrm{kHz}$ is detected, the frequency response of the transmitter (Figure 11) maybe affecting the signal and the $300 \mathrm{kHz}$ resonance frequency is then clearly detected by the sensor. The CMUT response is principally centered around the $530 \mathrm{kHz}$ excitation frequency but the resonance frequency of the $100 \mu \mathrm{m}$ membrane (at $450 \mathrm{kHz})$ is also detected.

Besides, Figure 12(a) and (c) give an idea of the impact of the $V_{D C}$ on the response of the CMUT sensor. The higher is the DC bias voltage $V_{D C}$, the higher is the electrical signal from the CMUT, as expected. However, the electrical performances with regard to this voltage are slightly lower than expected highlighting a non perfect sum of the elementary contributions of each cell. Indeed, to ensure the operation of CMUT arrays, the DC bias voltage is at most about $50 \%$ of the pull-in voltage. In this case, the static deflection of the membranes remains low in front of the electrostatic gaps. Thus, the amplitude of the detected electrical signal should be theoretically proportional to the DC bias voltage, which is not accurately shown by Figure 12(a) (i.e. an amplitude ratio about only 3.5 between 1.4 and $4.9 \mathrm{mV}$ compare to a voltage ratio of 6 between 10 and $60 V_{D C}$ ).

To go further, the other excitation frequencies are studied. Figure 13(a) presents the FFTs obtained by the $\mu 80$ sensor and Figure 13(b) those obtained by the CMUT sensor with $60 V_{D C}$. The three excitation frequencies are compared: it can be observed that the amplitude of the $\mu 80$ sensor responses is really sensitive to the excitation frequency. All frequencies can effectively be captured (the scale can be misleading here but Figure 12(d) shows that the $530 \mathrm{kHz}$-frequency is well measured), but the amplitude ratio is highly variable according to the excitation frequency and could lead to misinterpretation in a data analysis. On the contrary, the CMUT sensor is more stable over the frequency band tested in this study. As for the $\mu 80$ sensor, the CMUT reacts more at its resonance frequency, but for the CMUT 

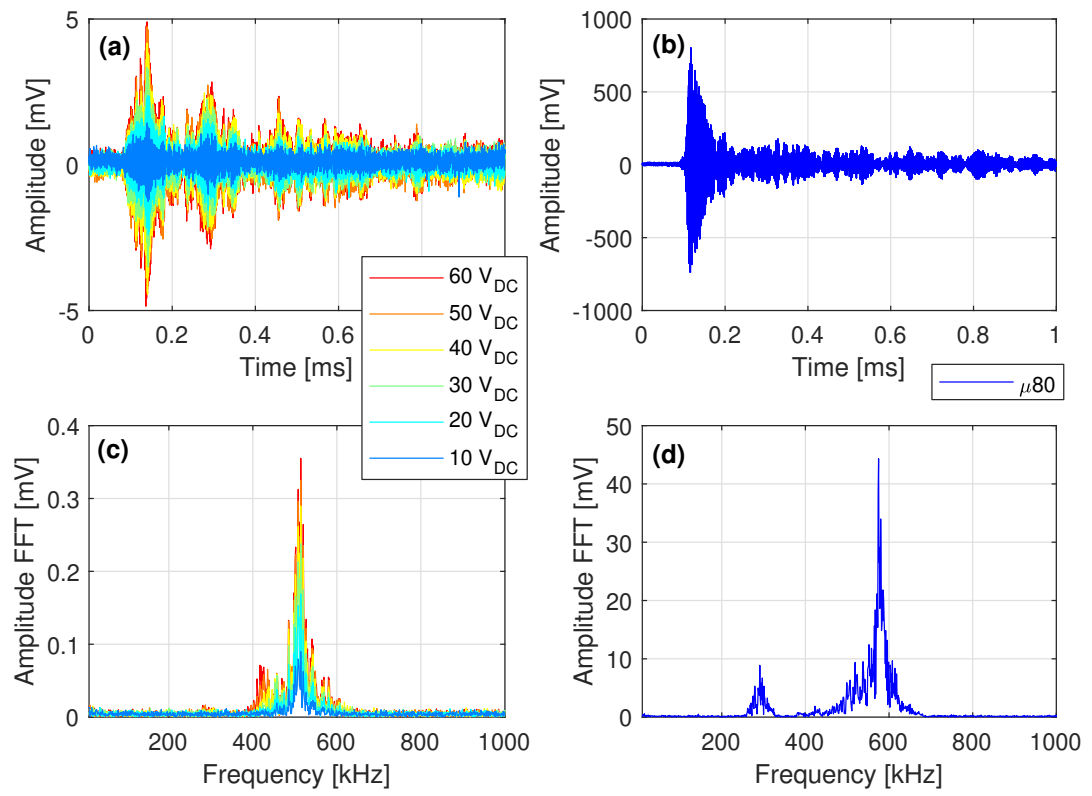

Figure 12: Experimental results at $530 \mathrm{kHz}$. (a) and (b) are the temporal signals measured respectively by the CMUT and the $\mu 80$. (c) and (d) are the FFT of the temporal signals. For the CMUT, the influence of the $V_{D C}$ is represented.

sensor, all frequencies are detected in a ratio of 1 to 3 , as opposed to a ratio of 1 to 45 for the $\mu 80$ sensor.

This analysis has also been performed for several bias voltages. The Signal-to-Noise Ratio (SNR), defined as

$$
S N R=10 \times \log _{10}\left(\frac{U_{S}}{U_{N}}\right)^{2},
$$

with $U_{S}$ the maximum amplitude measured on the temporal signal and $U_{N}$ the amplitude of the noise measured by the sensor, has been estimated for both sensors (Figure 14). Each sensor obviously has a better SNR at their resonance frequency. As expected, the commercial sensor $\mu 80$ has a much higher SNR than the CMUT, even with the highest bias voltage considered in this study. Nevertheless, as previously it can be observed that the SNR is varying according to the excitation frequency: the SNR of the CMUT is more stable (a variation amplitude of $10 \mathrm{~dB}$ compared to $20 \mathrm{~dB}$ for the $\mu 80)$. Finally, the CMUT, even far away from the commercial sensor in terms 
(a) $\mu 80$ measurements

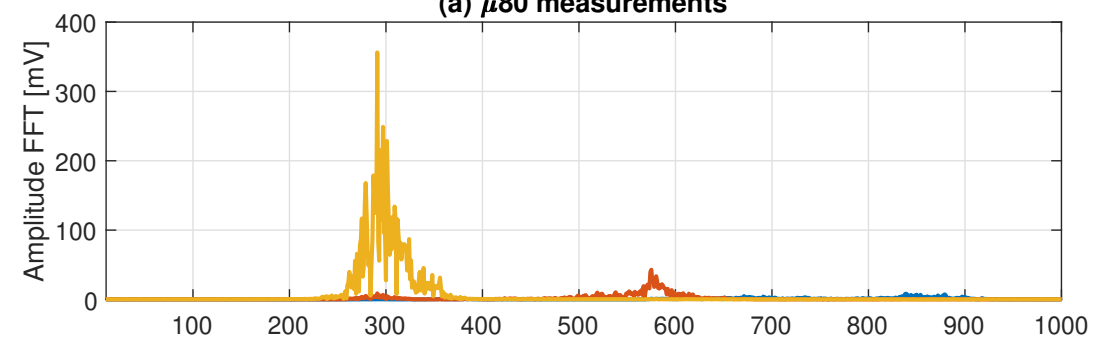

(b) CMUT measurements

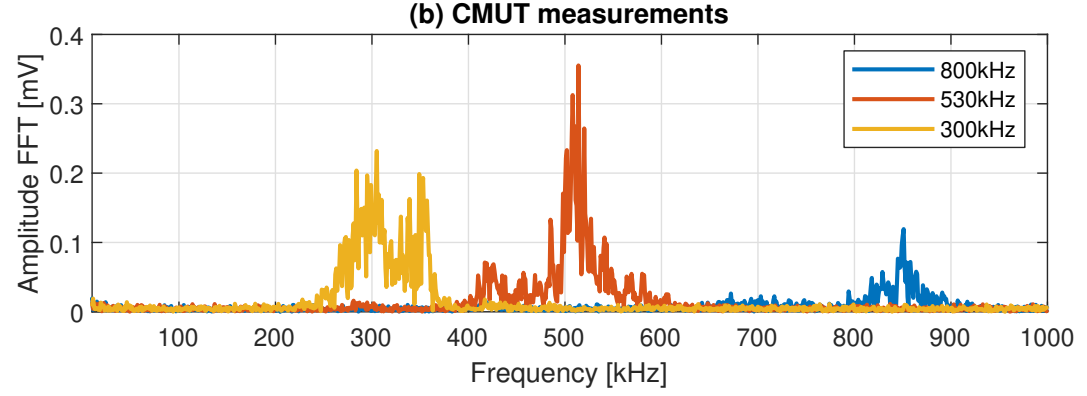

Figure 13: FFT of the temporal signals (a) for the $\mu 80$ sensor, used as a reference and (b) for the CMUT with $60 V_{D C}$ - for the different excitation frequencies.

of SNR, seems promising especially since the CMUT sensor sensitivity can still be improved: the numbers of membrane can easily be increased, the packaging should be enhanced with regard to acoustic waves energy transfer and parasitic noises, the elementary contribution of each cell and the efficient sum of all of them need to be further analyzed, etc.

To conclude on the results of harmonic excitation, arrays of identical CMUT membranes can be sensitive to a broad range of frequencies below and beyond the resonance frequency of the elementary cell.

The next section consists of the presentation of a classical test case in the field of Acoustic Emission in order to highlight possible capabilities of CMUT sensors in SHM applications. 


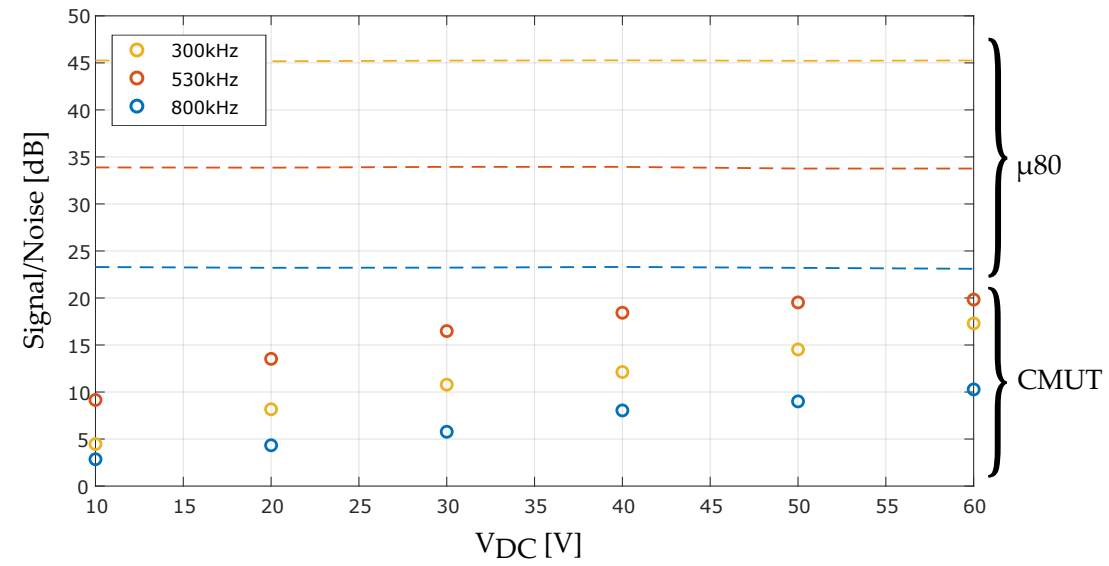

Figure 14: Signal-to-Noise Ratio of the $\mu 80$ (dashed lines) and of the CMUT (dot points) at $300 \mathrm{kHz}, 530 \mathrm{kHz}$ and $800 \mathrm{kHz}$, according to the bias voltage.

\subsection{Acoustic emission test simulation}

In this section we use an artificial source commonly used in acoustic emission: the Pencil Lead Break (PLB) [25] which allows to generate a broadband excitation.

\subsubsection{Materials and method}

In this configuration, to avoid rigid body motion, the aluminium beam is clamped at one end (Figure 15). The $2 \mathrm{H}$ lead has a $0.5 \mathrm{~mm}$ diameter, and it is broken with a length of about $2 \mathrm{~mm}$. At the other end of the beam, spring clamps are used to attach the two sensors, the CMUT and the $\mu 80$. As previously, array of 9 membranes of $100 \mu \mathrm{m}$ radius is involved.

\subsubsection{Results - broadband excitation}

The temporal signal and the FFT of the signals are studied for both sensors on the Figure 16. A higher magnitude on the FFT, centered around $300 \mathrm{kHz}$ for the $\mu 80$, and on $450 \mathrm{kHz}$ for the CMUT can be observed. These frequencies correspond to the resonance frequencies of the sensors. This kind of excitation then permits to highlight the footprint of the sensor.

The SNR is not post-processed here, but as it can be seen on the Figure 16(a) the temporal signal of the CMUT sensor is quite noisy. The noise, before and after the PLB test, is around $0.4 \mathrm{mV}$. With the highest bias voltage studied of $60 \mathrm{~V}$ the maximum amplitude observed is $1.3 \mathrm{mV}$. It is 


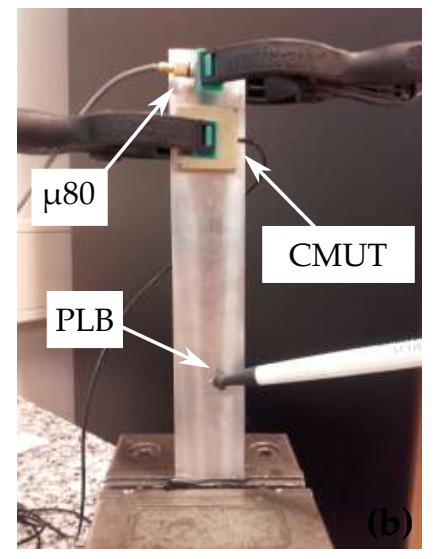

Figure 15: Experimental setup for pencil lead break excitation.

quite small but it still allows the CMUT to pick up the event. The more the bias voltage decreases the more difficult it is to get out of the noise. However, the FFT of the signals reported on Figure 16(c) shows several amplitude peaks from a DC bias voltage of $40 \mathrm{~V}$ and more pronounced peaks beyond that up to $60 \mathrm{~V}$ in our study. This richness of the frequency spectrum remains to be studied in more details in order to discriminate their real meaning. This could be advantageously used in data processing methods required for the SHM process. 

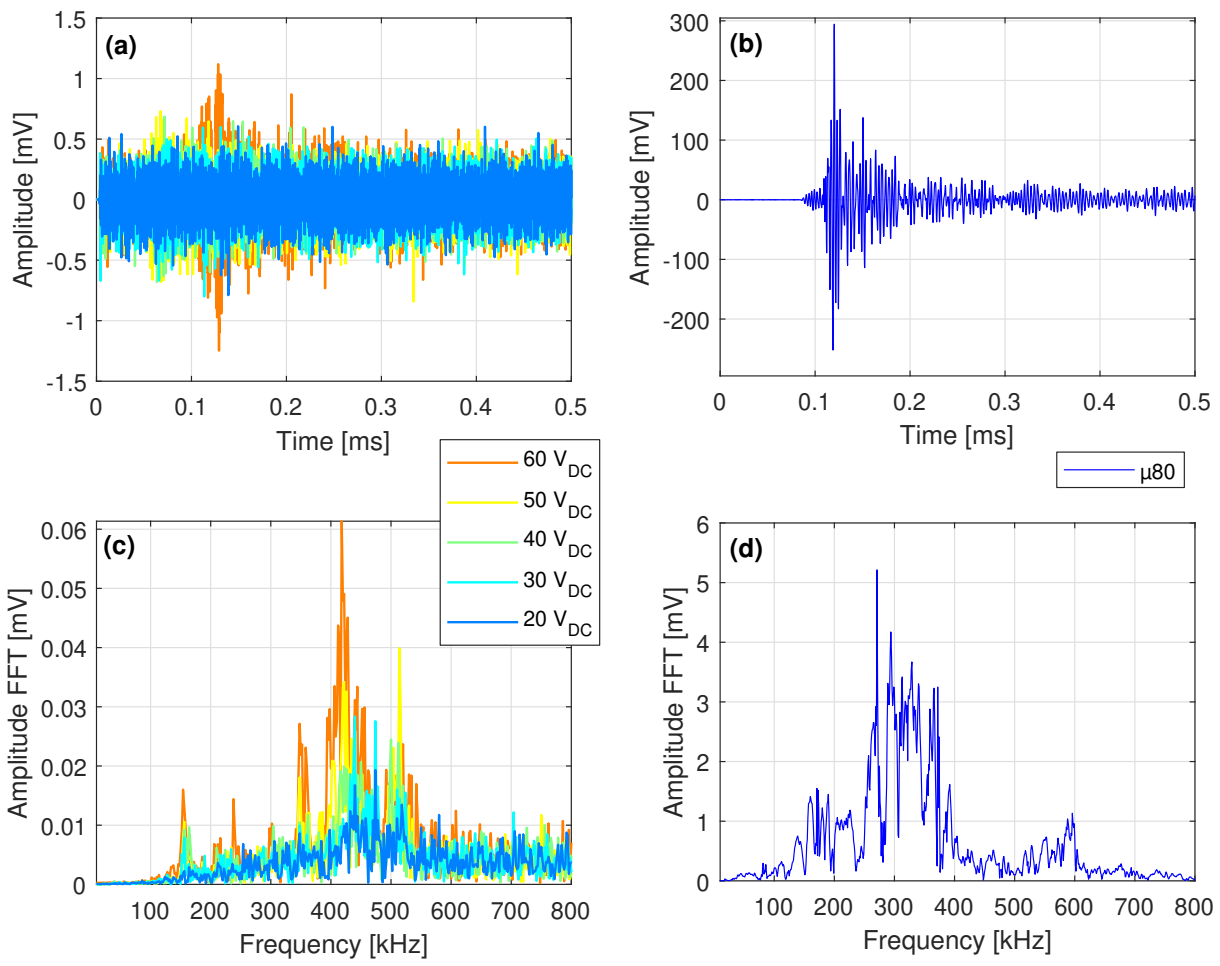

Figure 16: Response to a pencil lead break excitation. (a) and (b) are the temporal signals measured respectively by the CMUT and the $\mu 80$. (c) and (d) are the FFT of the temporal signals. For the CMUT, the influence of the $V_{D C}$ is represented.

\section{Conclusion and perspectives}

A CMUT sensor based on circular membranes is developed and characterized. A design procedure is proposed to obtain a sensor sensitive over a wide range of target frequencies.

Two aspects are considered: the multi-frequency aspect which involves different individual circular membranes (from $50 \mu \mathrm{m}$ to $250 \mu \mathrm{m}$ radius) and the bandwidth aspect which concerns the intrinsic capabilities of an array of 9 identical membranes of $100 \mu \mathrm{m}$ radius. An analytical approach is used to determine the frequency range of the CMUT: between $80 \mathrm{kHz}$ and $2 \mathrm{MHz}$ according to the radius. A numerical study is then developed which highlights the geometrical parameters influencing the electromechanical behaviour of a CMUT cell. In particular, the stepped geometry of the membrane leads to a consequent stiffening of the CMUT and a significant shift of the resonance 
frequency. Besides, this study involves a perforated membrane and it appears that the releasing holes induce a quite significant reduction of the stiffness of the membrane. These geometric features should then be taken into account when dimensioning this kind of cell.

In a second step, an exhaustive experimental study is performed. Taking into account realistic residual stresses, a good correlation is obtained between the resonance frequencies of the numerical model and the experimental measurements for moderate slenderness membranes. However, for membranes with a slenderness ratio greater than 150, buckling phenomena induced by residual stresses seem to modify the dynamic properties of the structure leading to an increase of the resonant frequencies. Besides, an electromechanical characterization of a CMUT array of identical membranes highlights the sensitivity of the CMUT according to the frequency excitation and for different bias voltages. The signal to noise ratio of the CMUT is fully dependent on the excitation frequency (higher when closer to the resonance frequency) and can be improved by increasing the bias voltage. Finally the CMUT sensitivity of the array studied is still far away from commercial sensors but seems very promising in terms of frequency range.

Lastly, a broadband characterization is done thanks to a pencil lead break which highlights the ability of the CMUT array to detect acoustic emission events. The influence of the bias voltage on the CMUT sensitivity is again clearly demonstrated.

Thus, CMUT sensors reveals actual capabilities in the targeted SHM application from both multi-frequency and bandwidth aspects considered separately in the present study.

A next step in this work is to combine these two aspects by testing membrane arrays with different radii in order to further enhance the frequency band covered. In addition to the problems associated with the various possible optimal bias voltages, a compromise will have to be found, in terms of the sensor footprint, between frequency bandwidth and sensitivity.

Therefore, the implementation of CMUT sensors in real situations will also require further theoretical and experimental works on the electromechanical parameters influencing the ability of an elementary cell to convert dynamic mechanical energy into electrical energy, on the optimization of the transfer of acoustic energy from the monitored structure to the elementary cell and on noise reduction. All these possible ways of improving the signal-to-noise ratio are the next challenges for the success of CMUT sensors. 


\section{Acknowledgment}

This works was supported by Bourgogne Franche-Comté Region in collaboration with EUR EIPHI Graduate School (contract ANR-17-EURE-0002). This work was partly supported by the french RENATECH network and its FEMTO-ST technological facility.

\section{References}

[1] M. Saeedifar, D. Zarouchas, Damage characterization of laminated composites using acoustic emission: A review, Composites Part B: Engineering (2020) 108039.

[2] I. García, J. Zubia, G. Durana, G. Aldabaldetreku, M. Illarramendi, J. Villatoro, Optical fiber sensors for aircraft structural health monitoring, Sensors 15 (7) (2015) 15494-15519.

[3] K. Bremer, F. Weigand, Y. Zheng, L. Alwis, R. Helbig, B. Roth, Structural health monitoring using textile reinforcement structures with integrated optical fiber sensors, Sensors 17 (2) (2017) 345.

[4] N. Chandarana, D. Sanchez, C. Soutis, M. Gresil, Early damage detection in composites during fabrication and mechanical testing, Materials $10(7)$ (2017) 685.

[5] J. Jung, W. Lee, W. Kang, E. Shin, J. Ryu, H. Choi, Review of piezoelectric micromachined ultrasonic transducers and their applications, Journal of Micromechanics and Microengineering 27 (11) (2017) 113001.

[6] I. J. Oppenheim, A. Jain, D. W. Greve, Mems ultrasonic transducers for the testing of solids, IEEE transactions on ultrasonics, ferroelectrics, and frequency control 50 (3) (2003) 305-311.

[7] I. J. Oppenheim, A. Jain, D. W. Greve, Electrical characterization of coupled and uncoupled mems ultrasonic transducers, IEEE Transactions on Ultrasonics, Ferroelectrics, and Frequency Control 50 (3) (2003) 297304.

[8] A. Galisultanov, P. Le Moal, G. Bourbon, V. Walter, Squeeze film damping and stiffening in circular cmut with air-filled cavity: Influence of the 
lateral venting boundary conditions and the bias voltage, Sensors and Actuators A: Physical 266 (2017) 15-23.

[9] D. Ozevin, D. Greve, I. Oppenheim, S. Pessiki, Resonant capacitive mems acoustic emission transducers, Smart materials and structures 15 (6) (2006) 1863.

[10] D. A. Koester, R. Mahadevan, B. Hardy, K. W. Markus, Mumps design handbook, Cronos Integrated Microsystems, Research Triangle Park, NC (2001) 1-41.

[11] D. W. Greve, I. J. Oppenheim, W. Wu, A. P. Wright, Development of a mems acoustic emission sensor system, in: Sensors and Smart Structures Technologies for Civil, Mechanical, and Aerospace Systems 2007, Vol. 6529, International Society for Optics and Photonics, 2007, p. 652912.

[12] D. Hutchins, D. Billson, R. Bradley, K. Ho, Structural health monitoring using polymer-based capacitive micromachined ultrasonic transducers (cmuts), Ultrasonics 51 (8) (2011) 870-877.

[13] H. Saboonchi, D. Ozevin, Mems acoustic emission transducers designed with high aspect ratio geometry, Smart Materials and Structures 22 (9) (2013) 095006.

[14] M. Kabir, H. Kazari, D. Ozevin, Piezoelectric mems acoustic emission sensors, Sensors and Actuators A: Physical 279 (2018) 53 - 64.

[15] T. A. Emadi, D. A. Buchanan, A novel $6 \times 6$ element mems capacitive ultrasonic transducer with multiple moving membranes for high performance imaging applications, Sensors and Actuators A: Physical 222 (2015) 309-313.

[16] D. Koester, R. Mahadevan, B. Hardy, K. Markus, Multi-user mems processes (mumps) design handbook, Cronos Integrated Microsystems, JDS Uniphase, NC, Revision 6.

[17] D. C. Miller, B. L. Boyce, M. T. Dugger, T. E. Buchheit, K. Gall, Characteristics of a commercially available silicon-on-insulator mems material, Sensors and Actuators A: Physical 138 (1) (2007) 130-144. 
[18] S. Mohite, H. Kesari, V. Sonti, R. Pratap, Analytical solutions for the stiffness and damping coefficients of squeeze films in mems devices with perforated back plates, Journal of Micromechanics and Microengineering 15 (11) (2005) 2083.

[19] I. Crandall, The air-damped vibrating system: Theoretical calibration of the condenser transmitter, Physical Review 11 (6) (1918) 449.

[20] S. Ghaffari, E. J. Ng, C. H. Ahn, Y. Yang, S. Wang, V. A. Hong, T. W. Kenny, Accurate modeling of quality factor behavior of complex silicon mems resonators, Journal of Microelectromechanical Systems 24 (2) (2014) 276-288.

[21] Z. Hao, A. Erbil, F. Ayazi, An analytical model for support loss in micromachined beam resonators with in-plane flexural vibrations, Sensors and Actuators A: Physical 109 (1-2) (2003) 156-164.

[22] K. K. Park, H. J. Lee, P. Crisman, M. Kupnik, O. Oralkan, B. KhuriYakub, Optimum design of circular cmut membranes for high quality factor in air, in: 2008 IEEE Ultrasonics Symposium, IEEE, 2008, pp. 504-507.

[23] A. W. Leissa, Nasa sp-160, Vibration of plates (1969) 59-60.

[24] A. Nikoozadeh, B. Bayram, G. G. Yaralioglu, B. T. Khuri-Yakub, Analytical calculation of collapse voltage of cmut membrane [capacitive micromachined ultrasonic transducers], in: Ultrasonics Symposium, 2004 IEEE, Vol. 1, IEEE, 2004, pp. 256-259.

[25] M. G. Sause, Investigation of pencil-lead breaks as acoustic emission sources., Journal of acoustic emission 29. 